\title{
Ist Beratung eine sinnvolle Intervention bei Übergewicht und Adipositas?
}

Fragestellung: Systematische Übersichtsarbeit zur Wirksamkeit von Beratung bei übergewichtigen und adipösen Patienten in der allgemeinärztlichen Versorgung.

Hintergrund: Es gibt eine weltweite Epidemie von Übergewicht mit ungünstigen Folgen für Lebenserwartung, Lebensqualität, Entstehung und Verlauf von medizinischen Erkrankungen und psychischen Störungen. Bisherige Maßnahmen haben den Verlauf dieser Epidemie nicht abgeschwächt. Trotz der großen gesundheitspolitischen Bedeutung gibt es einen erheblichen Mangel an Wissen über die Wirksamkeit verhaltensbasierter Interventionen.

Patienten und Methodik: Es handelt sich um eine systematische Review aller randomisierten, kontrollierten Studien, die zwischen 1980 und 2014 pu-

Wadden TA, Butryn ML, Hong PS et al. Behavioral treatment of obesity in patients encountered in primary care settings. A Systematic Review. JAMA 2014; 312: 1779-91 bliziert wurden, übergewichtige oder adipöse Patienten aus der Primärversorgung rekrutiert haben und mit einer Beratungsintervention zu Ernährung, Sport und zugehörigen Verhaltensproble- men über mindestens drei Monate behandelt haben. Weitere Einschlusskriterien waren eine Nachbeobachtungszeit von mindestens sechs Monaten nach der Randomisierung, eine Mindestgruppengröße von 15 pro Behandlung, objektive Gewichtsbestimmung, das Vorhandensein einer Vergleichsgruppe, eine Intention-to-treat-Analyse und eine Abbrecherquote von unter $30 \%$.

Ergebnisse: Es wurden zwölf Studien mit insgesamt 3.893 Teilnehmern identifiziert. Der mittlere Ausgangswert des BodyMass-Index (BMI) lag zwischen 32 und $39 \mathrm{~kg} / \mathrm{m}^{2}$. In den Vergleichsgruppen reichte das Spektrum des Gewichtsverlaufs in sechs Monaten von einer mittleren Zunahme von $0,9 \mathrm{~kg}$ zu einer Abnahme von 2,0 kg, in den Interventionsgruppen lag das Spektrum zwischen einer mittleren Abnahme von $0,3 \mathrm{~kg}$ zu einer Abnahme von 6,6 kg. Interventionen, die gezielte Vorgaben zur Ernährung und zur körperlichen Aktivität machten und diese in ein Verhaltensmanagementprogramm einbauten, waren im Mittel deutlich erfolgreicher als Programme denen diese Elemente fehlten und die sich auf motivationale Faktoren beschränkten.

Schlussfolgerungen: Beratung kann zu einem klinisch relevanten Gewichtsverlust führen.

\section{- Kommentar von Ulrich Schweiger, Lübeck}

\section{In diesem Feld sind neue kreative Ideen gefragt}

Trotz der positiven Schlussfolgerung des Reviews sind die Ergebnisse ernüchternd. Zum einen zeigt die Studie, wie wenige qualitativ hochwertige Studien in diesem Bereich vorliegen. Zum anderen ließen sich auch in Studien mit sehr gutem Design und gut geschulten Interventionsteams nur Ergebnisse mit geringen Effektstärken erzielen. Dabei wurden harte Endpunkte wie kardiovaskuläre Morbidität oder Mortalität gar nicht erfasst. Zur Erinnerung: Die Look-AHEAD-Studie, eine mit 5.145 Teilnehmern riesige Studie mit adipösen Patienten mit Typ-2-Diabetes und einem sehr intensiven Interventionsprogramm, wurde 2013 bei einem mittleren Follow-up von 9,6 Jahren abgebrochen,

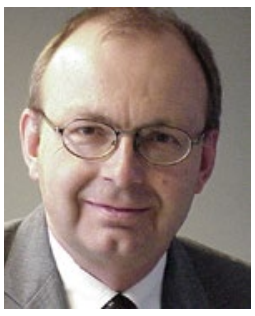

Prof. Dr. med. Ulrich Schweiger, Lübeck

Klinik für Psychiatrie und Psychotherapie, Universitätsklinikum Schleswig Holstein, Lübeck

E-Mail: Ulrich.Schweiger@uksh.de weil das Ziel, eine Reduktion von kardiovaskulären Ereignissen zu erreichen, als nicht mehr erreichbar befunden wurde. Deutlich bessere Ergebnisse finden sich, wenn bei Patienten, die noch nicht an Diabetes leiden, Sport und Ernährungsinterventionen zur Prävention eingesetzt werden. Ein interessantes Detail des Reviews von Wadden ist, dass Beratung, die sich auf die Förderung von Veränderungsmotivation beschränkt und keine konkreten Verhaltensvorgaben macht, nur sehr geringe Wirksamkeit aufweist. Die Behandlung von Übergewicht und Adipositas bleibt ein Feld, in dem neue kreative Ideen gefragt sind.

\section{Erratum}

In der Ausgabe 2/2015 (Seite 14) der InFo Neurologie \& Psychiatrie ist der Kommentar zum Artikel „Antidepressiva bei Kindern und Jugendlichen Induktion selbstschädigender Handlungen besonders unter hohen Dosierungen" durch einen technischen Fehler leider unvollständig abgedruckt worden. Der letzte Satz muss vollständig heißen "Leider scheitert dies in der Praxis oft daran, dass Therapieplätze nicht rasch genug verfügbar sind." Wir bitten um Entschuldigung. 